\title{
A SYSTEM OF BI-IDENTITIES FOR LOCALLY INVERSE SEMIGROUPS
}

\author{
K. AUINGER
}

(Communicated by Ronald M. Solomon)

\begin{abstract}
A class of regular semigroups closed under taking direct products, regular subsemigroups, and homomorphic images is an existence-variety (or $e$ variety) of regular semigroups. Each $e$-variety of locally inverse semigroups can be characterized by a set of bi-identities. These are identities of terms of type $\langle 2,2\rangle$ in two sorts of variables $X$ and $X^{\prime}$. In this paper we obtain a basis of bi-identities for the $e$-variety of locally inverse semigroups and for certain sub-e-varieties.
\end{abstract}

\section{INTRODUCTION}

In order to study regular semigroups from a "universal" perspective, Hall $[6,7,8]$ introduced the notion of existence variety (or $e$-variety) of regular semigroups: this is a class of regular semigroups closed under taking direct products, regular subsemigroups, and homomorphic images. He showed that the collection of all $e$-varieties of regular semigroups forms a complete lattice under inclusion (containing the lattices of inverse, respectively completely regular, semigroup varieties as ideal sublattices).

Independently, Kadourek and Szendrei [10] introduced the concept of $e$ variety for the class of orthodox semigroups, using the term "bivariety". In addition they showed that $e$-varieties of orthodox semigroups are determined by sets of bi-identities. In this context a bi-identity is a semigroup identity in two sorts of variables $x$ and $x^{\prime}$. A bi-identity $u=v$ is said to hold in an orthodox semigroup $S$ if each substitution of the variables in $u$ and $v$ by elements of $S$ in such a way that $x$ and $x^{\prime}$ are substituted by mutually inverse elements of $S$ yields the same element of $S$ on both sides. Kadourek and Szendrei have shown that the $e$-varieties of orthodox semigroups are precisely the classes consisting of all orthodox semigroups which satisfy a set of bi-identities. In this theory, the bifree object on a set $X$ seems to be as fundamental as the free object is for usual varieties of universal algebras. The definition, given by Kadourek and Szendrei for orthodox semigroups, can be formulated for arbitrary classes of regular semigroups.

Received by the editors October 20, 1992.

1991 Mathematics Subject Classification. Primary 20M07, 20 M17.

Financial support by "Fonds zur Förderung der wissenschaftlichen Forschung in Österreich" (No. P 8764-PHY) is gratefully acknowledged. 
Definition 1. Let $X$ be a non-empty set and $X^{\prime}=\left\{x^{\prime} \mid x \in X\right\}$ be a disjoint copy of $X, x \mapsto x^{\prime}$ being a bijection. Let $S$ be a regular semigroup. A mapping $\theta: X \cup X^{\prime} \rightarrow S$ is matched if $x^{\prime} \theta$ is an inverse of $x \theta$ in $S$ for each $x \in X$. Now let $\mathscr{V}$ be a class of regular semigroups, and $X$ and $X^{\prime}$ be as above. A semigroup $F \in \mathscr{V}$ together with a matched mapping $\imath: X \cup X^{\prime} \rightarrow F$ is a bifree object on $X$ in $\mathscr{V}$ if for any $S \in \mathscr{V}$ and any matched mapping $\theta: X \cup X^{\prime} \rightarrow S$ there is a unique homomorphism $\bar{\theta}: F \rightarrow S$ extending $\theta$, that is, satisfying $\imath \bar{\theta}=\theta$.

Kadourek and Szendrei have shown that in each $e$-variety $\mathscr{V}$ of orthodox semigroups, the bifree object $\mathrm{BF} \mathscr{V}(X)$ on any nonempty set $X$ exists and is up to isomorphism uniquely determined by the cardinality of $X$. These bifree objects behave in a way similar to the usual free objects in varieties of universal algebras. For instance, there is an order-inverting bijection between the lattice of all fully invariant congruences on the bifree orthodox semigroup $\mathrm{BF} \mathscr{O}(X)$ on a countably infinite set $X$ and the lattice of all $e$-varieties of orthodox semigroups. Also, each semigroup $S \in \mathscr{V}$ is a homomorphic image of some bifree object in $\mathscr{V}$. The bifree orthodox semigroup $\mathrm{BF} \mathscr{O}(X)$ on $X$ coincides with the free orthodox $*$-semigroup described by Szendrei [24].

For the nonorthodox case, Y. T. Yeh [26] has shown that all bifree objects exist in an $e$-variety $\mathscr{V}$ if and only if all members of $\mathscr{V}$ are either $E$-solid or locally inverse. In the latter case, the methods of [26] suggest that we introduce another binary operation $\wedge$ on a locally inverse semigroup $S$ and that we treat $S$ as an algebra of type $\langle 2,2\rangle$. This allows us to define the notion of biidentity for locally inverse semigroups (in analogy to the orthodox case [10]). In this context bi-identities are identities of terms of type $\langle 2,2\rangle$ in the variables $X \cup X^{\prime}$. Further, the bifree object $\operatorname{BF} \mathscr{V}(X)$ in the $e$-variety $\mathscr{V}$ on the set $X$ is isomorphic to $F_{\langle 2,2\rangle}\left(X \cup X^{\prime}\right) / \rho(\mathscr{V})$ where $F_{\langle 2,2\rangle}\left(X \cup X^{\prime}\right)$ is the absolutely free algebra of type $\langle 2,2\rangle$ generated by the set $X$ and the set of formal inverses $X^{\prime}$, and $\rho(\mathscr{V})$ is the congruence consisting of all pairs $(u, v)$ for which the bi-identity $u=v$ holds in $\mathscr{V}$. The word problem for the bifree locally inverse semigroup $\mathrm{BF} \mathscr{L} \mathscr{S}(X)$ on $X$ has been solved in [1]. That is, we have obtained an algorithm for deciding whether or not a bi-identity $u=v$ holds in the class $\mathscr{L} \mathscr{S}$ of all locally inverse semigroups.

The purpose of the present paper is to obtain bases of bi-identities for the $e$-variety $\mathscr{L} \mathscr{S}$ and for certain sub-e-varieties. We thus answer a question of M. Volkov raised at the Conference on Semigroups in Luino, italy, June 1992, where the author presented the results of [1]. This is the content of sections 3 and 4 . In section 2 we present some preliminary information.

\section{Preliminaries}

For undefined notions the reader is referred to the books of Grätzer [4], Howie [9], and Petrich [22]. We shall summarize some important facts about locally inverse semigroups.

For any semigroup $S$ let $E(S)$ denote the set of idempotents of $S$; for $x \in S$ let $V(x)=\{y \in S \mid x y x=x, y x y=y\}$ be the set of inverses of $x$ in $S$. Given two idempotents $e, f \in E(S)$ then $S(e, f)=f V(e f) e$ is the sandwich set of the pair $(e, f)$. If $S$ is regular, then for any $e, f \in E(S)$, the sandwich set $S(e, f)$ is not empty and consists of (some) idempotent inverses 
of $e f$. A regular semigroup $S$ is locally inverse if each local submonoid $e S e$ of $S, e \in E(S)$, is an inverse semigroup. These semigroups have been studied by several authors, see for instance Nambooripad [15-19] and Pastijn [20, 21]. It has been shown by Nambooripad [15, Theorem 7.6] that a regular semigroup $S$ is locally inverse if and only if each sandwich set $S(e, f), e, f \in E(S)$, consists of precisely one element. In any regular semigroup $S$, the sandwich set $S\left(a^{\prime} a, b b^{\prime}\right)$ is independent of the choice of the inverses $a^{\prime} \in V(a)$ and $b^{\prime} \in V(b)$ (see [25, 2.1.1]). This fact has been used by Yeh [26] to introduce another binary operation on any locally inverse semigroup.

Definition 2. Let $S$ be a locally inverse semigroup, $x, y \in S$ and $x^{\prime} \in V(x)$, $y^{\prime} \in V(y)$. Then $x \wedge y$ denotes the uniquely determined element in $S\left(y^{\prime} y, x x^{\prime}\right)$. The binary operation $(x, y) \mapsto x \wedge y$ will be called the sandwich operation.

The following important lemma has been proved by Yeh [26, Lemma 3.2.1]:

Lemma 2.1. Any semigroup homomorphism between two locally inverse semigroups respects the sandwich operation $\wedge$.

Let $X$ be an infinite set and $X^{\prime}=\left\{x^{\prime} \mid x \in X\right\}$ be a disjoint copy of $X$, $x \mapsto x^{\prime}$ being a bijection. Let $\left(F_{\langle 2,2\rangle}\left(X \cup X^{\prime}\right), \cdot, \wedge\right)$ denote the absolutely free algebra of type $\langle 2,2\rangle$ generated by the set $X \cup X^{\prime}$. In the following we consider locally inverse semigroups $S$ as algebras of type $\langle 2,2\rangle$.

Definition 3. Let $S$ be a locally inverse semigroup and $\phi: F_{\langle 2,2\rangle}\left(X \cup X^{\prime}\right) \rightarrow S$ be a homomorphism. Then $\phi$ is matched if $\phi \mid X \cup X^{\prime}$ is a matched mapping.

The notion of bi-identity for the case of locally inverse semigroups has been introduced in [1]. Notice that the binary operation $*$ in [1] is the dual of the binary operation $\wedge$ here.

Definition 4. Let $S$ be a locally inverse semigroup and $w, v \in F_{\langle 2,2\rangle}\left(X \cup X^{\prime}\right)$. Then the pair $w=v$ is a bi-identity. The bi-identity $w=v$ holds in $\mathrm{S}$ if $w \phi=v \phi$ for each matched homomorphism $\phi: F_{\langle 2,2\rangle}\left(X \cup X^{\prime}\right) \rightarrow S$. The biidentity $w=v$ holds in the class $\mathscr{V}$ of locally inverse semigroups if it holds in every member of $\mathscr{V}$.

Remark. We always will consider bi-identities as pairs of elements of $F_{\langle 2,2\rangle}\left(X \cup X^{\prime}\right)$, independently of whether they are expressed in the form $w=v$ or $(w, v)$. Therefore it is reasonable to speak about the equivalence relation, congruence relation, etc., which is generated by a given set of bi-identities $B$. In particular, each congruence on $F_{\langle 2,2\rangle}\left(X \cup X^{\prime}\right)$ is a specific set of bi-identities.

Recall that the class $\mathscr{L} \mathscr{F}$ of all locally inverse semigroups is an $e$-variety (see Nambooripad [16, Theorem 3.2]).

In [1] we have pointed out the following.

(1) A class $\mathscr{V}$ of locally inverse semigroups is an $e$-variety if and only if there is a set $\rho$ of bi-identities such that $\mathscr{V}$ consists precisely of all locally inverse semigroups $S$ which satisfy each bi-identity from $\rho: \mathscr{V}=\mathscr{V}(\rho)$.

(2) For each $e$-variety $\mathscr{V}$ of locally inverse semigroups the bifree object on $X$ in $\mathscr{V}$ is given by $F_{\langle 2,2\rangle}\left(X \cup X^{\prime}\right) / \rho(\mathscr{V})$ where $\rho(\mathscr{V})$ is the congruence consisting of all bi-identities which hold in $\mathscr{V}$.

In [1] it was convenient to define bi-identities to be pairs $(w, v)$ of elements of the absolutely free algebra $F_{\langle 2,2\rangle}\left(X \cup X^{\prime}\right)$ on the set $X \cup X^{\prime}$ in two binary 
operations $\cdot$ and $\wedge$. For the present purpose it is more convenient to assume that the multiplication - in $F_{\langle 2,2\rangle}\left(X \cup X^{\prime}\right)$ satisfies the associative law (and will be expressed simply by concatenation). Of course, all mentioned results stay true if we assume that the multiplication - is associative. Therefore, from now on, let $F=F_{\langle 2,2\rangle}\left(X \cup X^{\prime}\right)$ be the free algebra on $X \cup X^{\prime}$ in two binary operations - and $\Lambda$, the former one being associative. Shortly we shall call an algebra $(S, \cdot, \wedge)$ of type $\langle 2,2\rangle$ such that $\cdot$ is associative a "binary semigroup". As for the free unary semigroup (see for instance Clifford [3]), the free binary semigroup $F$ on the set $X \cup X^{\prime}$ can be described as follows.

Theorem 2.2. Let $(, \wedge$,$) be three distinct symbols (called l-par, wedge, and r$ par) which are not contained in $X \cup X^{\prime}$. Let $S$ be the free semigroup on the alphabet $X \cup X^{\prime} \cup\{(, \wedge)$,$\} . Let G \subseteq S$ be the smallest subset of $S$ such that:

(1) $X \cup X^{\prime} \subseteq G$,

(2) $u, v \in G \Rightarrow u v \in G,(u \wedge v) \in G$.

Then $G$ is a subsemigroup of $S$, equipped with an additional binary operation $\wedge:[u, v] \mapsto(u \wedge v)$. Furthermore, the algebra $(G, \cdot, \wedge)$ is a free binary semigroup on the set $X \cup X^{\prime}$.

Throughout we shall use [ , ] whenever it seems to be important to distinguish clearly between brackets and $l$-, respectively, $r$-pars $($,$) . Theorem 2.2$ can be proved similarly to the well-known description of the free unary semigroup [3]; the proof is therefore omitted. Also, one can prove that each element of $G$ admits a unique factorization into indecomposable elements. The latter are precisely the letters of $X \cup X^{\prime}$ and the elements of the form $w=(v \wedge u)$ where $v, u \in G$. For instance, $x,(x \wedge y)$, and $(x y \wedge(x \wedge y))$ are indecomposable whereas $x y$ decomposes into $x$ and $y$. Throughout the paper we shall identify $F$ with the semigroup $G$ of Theorem 2.2.

\section{A SYSTEM OF BI-IDENTITIES FOR LOCALLY INVERSE SEMIGROUPS}

For a countably infinite set $X$ let $F=F_{\langle 2,2\rangle}\left(X \cup X^{\prime}\right)$ be the free binary semigroup on $X \cup X^{\prime}$ as it is presented in Theorem 2.2. Let $\rho(\mathscr{L} \mathscr{S})$ be the set of all bi-identities which hold in $\mathscr{L S F}^{\mathcal{F}}$. In order to define the notion of basis of bi-identities for $\mathscr{L} \mathscr{S}$ we first give a (modified) definition of the notion of bi-invariant congruence on $F$. (Congruences on $F$ are assumed to respect both binary operations $\cdot$ and $\wedge$.) For terms $w, p, q \in F$ denote by $w(x \rightarrow$ $\left.p, x^{\prime} \rightarrow q\right)$ the term which is obtained by substituting each $x$ in $w$ by $p$ and each $x^{\prime}$ by $q$.

Definition 5. A congruence $\rho$ on $F$ is bi-invariant if:

(1) $x \rho x x^{\prime} x, x^{\prime} \rho x^{\prime} x x^{\prime}$ for all $x \in X$,

(2) $u \rho v, p \rho p q p$, and $q \rho q p q$ imply $u\left(x \rightarrow p, x^{\prime} \rightarrow q\right) \rho v(x \rightarrow$ $\left.p, x^{\prime} \rightarrow q\right)$.

Notice that in [1] and in [10] the definition of a bi-invariant congruence is different from the definition given here. First, in [1] (resp. [10]), for a congruence $\rho$ to be bi-invariant it is in addition required that $\rho(\mathscr{L} \mathscr{S}) \subseteq \rho$ (resp. $\rho(\mathscr{O}) \subseteq \rho$ in [10]). But also for congruences $\rho$ which contain $\rho(\mathscr{L} \mathscr{S})$, the definition here is prima facie different. By the definition in [1] respectively [10], substitution of $x$ and $x^{\prime}$ is only allowed if the terms $p$ and $q$ to be 
substituted for $x$, respectively $x^{\prime}$, are representatives of mutual inverses in $F / \rho(\mathscr{L} \mathcal{S})$ rather than $F / \rho$. Further, "simultaneous" substitution is allowed in [1] respectively [10]. However, by Result 1.1 in [10] (see [5]) it follows that if $p \rho$ and $q \rho$ are mutual inverses in $F / \rho$ for some $\rho$ containing $\rho(\mathscr{L} \mathscr{F})$, then there are $p_{1} \in p \rho$ and $q_{1} \in q \rho$ such that $p_{1} \rho(\mathscr{L} \mathscr{S})$ and $q_{1} \rho(\mathscr{L} \mathcal{F})$ are mutual inverses in $F / \rho(\mathscr{L} \mathcal{F})$. On the other hand, for bi-invariant congruences $\rho$ defined as above "simultaneous" substitution (in the sense of [1] respectively [10]) is allowed if $X$ is infinite. In fact, for congruences $\rho$ which contain $\rho(\mathscr{L} \mathcal{S})$ the definitions of being bi-invariant in [1] and here are equivalent. In particular, the bi-invariant congruences containing $\rho(\mathscr{L} \mathcal{F})$ are in a bijective correspondence to the $e$-varieties of locally inverse semigroups (see [1, Theorem 6.6]). Now the definition of a basis of bi-identities for a class $\mathscr{V}$ of locally inverse semigroups is the following.

Definition 6. Any set $B \subseteq F \times F$ of bi-identities which generates $\rho(\mathscr{V})$ as a bi-invariant congruence is a basis for the bi-identities of $\mathscr{V}$.

Let $B$ be a set of bi-identities. The bi-invariant congruence $\bar{B}$ generated by $B$ can be constructed as follows. For any set $B$ of bi-identities let $B^{\prime}$ consist of the following:

(A) all trivial bi-identities $w=w$ and all bi-identities $x=x x^{\prime} x, x^{\prime}=$ $x^{\prime} x x^{\prime}$ are in $B^{\prime}$

(B) if $u=v$ is in $B$, then $u=v, v=u$ are in $B^{\prime}$;

(C) if $u=v, v=w$ are in $B$, then $u=w$ is in $B^{\prime}$;

(D) if $u=v$ is in $B$, then for each $w \in F$ the bi-identities $w u=$ $w v, u w=v w,(w \wedge u)=(w \wedge v),(u \wedge w)=(v \wedge w)$ are all in $B^{\prime}$

(E) if $u=v, p=p q p, q=q p q$ are in $B$, then the bi-identity $u\left(x \rightarrow p, x^{\prime} \rightarrow q\right)=v\left(x \rightarrow p, x^{\prime} \rightarrow q\right)$ is in $B^{\prime}$.

Put $B_{0}=B, B_{1}=B^{\prime}$, and $B_{n}=B_{n-1}^{\prime}$ for each $n \in \mathbb{N}$. Then $\bar{B}=\bigcup_{n=1}^{\infty} B_{n}$ is the set of bi-identities which can be "obtained" from the set $B$. Likewise, $\bar{B}$ is the bi-invariant congruence generated by $B$.

Lemma 3.1. The congruence $\rho(\mathscr{L} \mathscr{S})$ is the least congruence $\rho$ on $F$ such that:

(1) $(F / \rho, \cdot)$ is a locally inverse semigroup,

(2) $x \rho$ and $x^{\prime} \rho$ are mutual inverses for all $x \in X$,

(3) the sandwich operation in $F / \rho$ is given by $(u \rho, v \rho) \mapsto(u \wedge v) \rho$.

Proof. The congruence $\rho(\mathscr{L} \mathscr{S})$ satisfies the conditions (1)-(3). Conversely, let $\rho$ be a congruence satisfying (1)-(3). The mapping $X \cup X^{\prime} \rightarrow F / \rho, x \mapsto$ $x \rho, x^{\prime} \mapsto x^{\prime} \rho$ is matched. Hence there is a unique homomorphism $\phi$ : $B F \mathscr{L} \mathcal{F}(X)=F / \rho(\mathscr{L} \mathscr{F}) \rightarrow F / \rho$ satisfying $x \rho(\mathscr{L} \mathscr{F}) \phi=x \rho$ and $x^{\prime} \rho(\mathscr{L} \mathscr{F}) \phi=$ $x^{\prime} \rho$. Clearly we have $[u v] \rho(\mathscr{L} \mathscr{F}) \phi=(u \rho(\mathscr{L} \mathscr{S}) \phi)(v \rho(\mathscr{L} \mathscr{S}) \phi)$. Let $\wedge$ denote the sandwich operation in $F / \rho$ as well as in $F / \rho(\mathscr{L} \mathscr{F})$. Then

$$
(u \wedge v) \rho(\mathscr{L} \mathscr{F}) \phi=(u \rho(\mathscr{L} \mathscr{F}) \wedge v \rho(\mathscr{L} \mathscr{F})) \phi=u \rho(\mathscr{L} \mathscr{F}) \phi \wedge v \rho(\mathscr{L} \mathscr{F}) \phi
$$

for all $u, v \in F$. Since $[u v] \rho=(u \rho)(v \rho)$ and $(u \wedge v) \rho=u \rho \wedge v \rho$ for all $u, v \in$ $F$, it follows by induction on the length of the term $u$ that $u \rho(\mathscr{L} \mathscr{F}) \phi=u \rho$ for all $u \in F$. In particular, $\rho(\mathscr{L} \mathscr{F}) \subseteq \rho$.

Remark. (3) cannot be omitted: let $\rho$ be the congruence generated by all pairs $\left(x, x^{\prime}\right), x \in X$, and all pairs $(u v, v u),(u, u u), u, v \in F$. Then $F / \rho$ is a 
semilattice such that $x \rho x^{\prime}$. Furthermore, $(u \rho, v \rho) \mapsto(u \wedge v) \rho$ is a welldefined binary operation on $F / \rho$ since $\rho$ respects $\wedge$. However, this operation does not coincide with the sandwich operation on $F / \rho$ since $[x y] \rho \neq(x \wedge y) \rho$ (for arbitrary $x, y \in X \cup X^{\prime}$ ) whereas the multiplication and the sandwich operation on any semilattice coincide. Hence $\rho$ satisfies (1) and (2), but it does not satisfy (3).

We are ready to formulate the main result.

Theorem 3.2. The bi-identities (1)-(4) form a basis for $\rho(\mathscr{L} \mathscr{F})$ :

(1) $(x \wedge y)=x x^{\prime}(x \wedge y) y^{\prime} y$,

(2) $(x \wedge y)=(x \wedge y)(x \wedge y)$,

(3) $y x=y(x \wedge y) x$,

(4) $\left(z x z^{\prime} \wedge z x z^{\prime}\right)\left(z y z^{\prime} \wedge z y z^{\prime}\right)=\left(z y z^{\prime} \wedge z y z^{\prime}\right)\left(z x z^{\prime} \wedge z x z^{\prime}\right)$.

Proof. The bi-identities (1)-(3) express well-known facts about elements in the sandwich set $S\left(y^{\prime} y, x x^{\prime}\right)$ (in any regular semigroup; see, e.g., Nambooripad [15]) and therefore hold in $\mathscr{L} \mathscr{S}$. Moreover, the element $z x z^{\prime} \wedge z x z^{\prime}$ stands for an arbitrary idempotent in the local submonoid $z z^{\prime} S z z^{\prime}=z S z^{\prime}$, and any two such idempotents commute in any locally inverse semigroup. Whence (4) holds in $\mathscr{L} \mathscr{S}$.

Let $\rho$ denote the bi-invariant congruence generated by all bi-identities (1)(4). We have thus shown that $\rho \subseteq \rho(\mathscr{L} \mathscr{S})$. In order to prove the reverse inclusion we first show that the bi-identities (1)-(3) imply the following ones:

(a) $(x \wedge y)=(x \wedge y) y^{\prime} y x x^{\prime}(x \wedge y)$,

(b) $y^{\prime} y x x^{\prime}=y^{\prime} y x x^{\prime}(x \wedge y) y^{\prime} y x x^{\prime}$,

(c) $y x=y x x^{\prime}(x \wedge y) y^{\prime} y x$

(d) $x^{\prime}(x \wedge y) y^{\prime}=x^{\prime}(x \wedge y) y^{\prime} y x x^{\prime}(x \wedge y) y^{\prime}$.

Using $x x^{\prime}=x x^{\prime} x x^{\prime}$ and $y^{\prime} y=y^{\prime} y y^{\prime} y$, by (1) we obtain the two bi-identities $(x \wedge y)=x x^{\prime}(x \wedge y)=(x \wedge y) y^{\prime} y$. By $(2)$ we get $(x \wedge y)=(x \wedge y)(x \wedge y)=$ $(x \wedge y) y^{\prime} y x x^{\prime}(x \wedge y)$, that is, (a). By (1) and (3) we get $y x=y x x^{\prime}(x \wedge y) y^{\prime} y x$, that is, (c). Multiplying (c) by $y^{\prime}$ on the left and $x^{\prime}$ on the right yields (b). Finally, multiplying (a) by $x^{\prime}$ on the left and $y^{\prime}$ on the right yields (d). Let $u, v, \tilde{u}, \tilde{v} \in F$, and suppose that $u \rho$ and $\tilde{u} \rho$ as well as $v \rho$ and $\tilde{v} \rho$ are mutually inverse elements in $F / \rho$. Then the bi-identities (a)-(d) imply that [uv] $\rho$ and $(u \wedge v) \rho$ have inverse elements in $F / \rho$. Since $x \rho$ and $x^{\prime} \rho$ are mutual inverses in $F / \rho$ and since $F / \rho$ is generated by the set $\left\{x \rho, x^{\prime} \rho \mid x \in\right.$ $X$ \} under the application of the operations - and $\wedge$, we infer that each element of $F / \rho$ admits an inverse. That is, $F / \rho$ is a regular semigroup. Next, let $w, v, \tilde{w}, \tilde{v} \in F$ be such that $w \rho$ (resp. $v \rho$ ) and $\tilde{w} \rho$ (resp. $\tilde{v} \rho$ ) are mutually inverse elements. By (a) and (b) we have $w \rho \wedge v \rho \in V((\tilde{v} \rho)(v \rho)(w \rho)(\tilde{w} \rho))$; thus by $(1), w \rho \wedge v \rho \in(w \rho)(\tilde{w} \rho) V((\tilde{v} \rho)(v \rho)(w \rho)(\tilde{w} \rho))(\tilde{v} \rho)(v \rho)$. In particular we get that

$$
w \rho \wedge v \rho \in S((\tilde{v} \rho)(v \rho),(w \rho)(\tilde{w} \rho)) .
$$

Finally let $w \in F$ be such that $w \rho w w$ (that is, the bi-identity $w=w w$ can be obtained from the system (1)-(4)). We obtain the bi-identity $w=w w w$. Hence in bi-identity (1) we may substitute $w$ for $x, y, x^{\prime}, y^{\prime}$ and obtain $(w \wedge$ $w)=w w(w \wedge w) w w=w(w \wedge w) w=($ by $(3))=w w=w$. Therefore, the system (1)-(4) implies the implication $w=w w \Rightarrow w=(w \wedge w)$ (for arbitrary 
$w \in F)$. We proceed to show that $S=F / \rho$ is locally inverse. Each local submonoid of $S$ is of the form $(w \rho)(\tilde{w} \rho) S(w \rho)(\tilde{w} \rho)=(w \rho) S(\tilde{w} \rho)$ for any $w, \tilde{w} \in F$ such that $w \rho$ and $\tilde{w} \rho$ are mutually inverse elements in $S$. Each monoid $(w \rho) S(\tilde{w} \rho)$ is clearly regular. Let $(w \rho)(v \rho)(\tilde{w} \rho)$ and $(w \rho)(u \rho)(\tilde{w} \rho)$ be idempotents of $(w \rho) S(\tilde{w} \rho)$. From the implication $p=p p \Rightarrow p=(p \wedge p)$ mentioned above we get $[w v \tilde{w}] \rho=(w v \tilde{w} \wedge w v \tilde{w}) \rho$ and likewise, $[w u \tilde{w}] \rho=$ $(w u \tilde{w} \wedge w u \tilde{w}) \rho$. Applying the bi-identity (4) we infer that the idempotents $(w \rho)(v \rho)(\tilde{w} \rho)$ and $(w \rho)(u \rho)(\tilde{w} \rho)$ commute so that $S$ is locally inverse. From $\left.{ }^{*}\right)$ we see that $[u \rho, v \rho] \mapsto u \rho \wedge v \rho$ is just the sandwich operation in $F / \rho$. By Lemma 3.1, $\rho(\mathscr{L} \mathscr{\mathscr { S }}) \subseteq \rho$ so that $\rho=\rho(\mathscr{L} \mathscr{F})$.

Remark. Within the system (1)-(4) of Theorem 3.2, the bi-identities (1), (2) may be replaced by the single bi-identity

$$
(x \wedge y)=x x^{\prime}(x \wedge y)(x \wedge y) y^{\prime} y .
$$

In the following we shall prove that the system of bi-identities (1)-(4) of Theorem 3.2 is independent. These bi-identities will be shortly referred to as the bi-identities (1)-(3), (1), (4), etc. Notice that in order to prove that biidentity $(j)$ is independent of the other ones it is sufficient to construct a binary semigroup $S$ generated by pairs of mutually inverse elements $x, x^{\prime}$ which does not satisfy bi-identity $(j)$ but does satisfy the other ones.

Lemma 3.3. The bi-identity (1) is independent of (2)-(4).

Proof. Let $B$ be the system of bi-identities (2)-(4). Each bi-identity $u=v \in B$ has the following property: the first respectively the last symbols in $u$ and $v$ are the same (either the same letter from $X \cup X^{\prime}$ or an $l$-par respectively an $r$ par). Application of the rules $(A)-(E)$ now again only yields bi-identities having this property. Consequently, (1) is not in $\bar{B}$.

Lemma 3.4. The bi-identity (2) is independent of (1), (3)-(4).

Proof. Let $B_{2}=\left\langle x, x^{\prime} \mid x=x x^{\prime} x, x^{\prime}=x^{\prime} x x^{\prime}, x x=0=x^{\prime} x^{\prime}\right\rangle$ be the 5element combinatorial Brandt semigroup. Define a binary operation $*$ by $x * x=x, x^{\prime} * x^{\prime}=x^{\prime}$, and $a * b=a \wedge b$ for all $(a, b) \notin\left\{(x, x),\left(x^{\prime}, x^{\prime}\right)\right\}$ where $\wedge$ denotes the sandwich operation. Then the binary semigroup $\left(B_{2}, \cdot, *\right)$ satisfies the bi-identities (1), (3)-(4), but it does not satisfy bi-identity (2).

Lemma 3.5. The bi-identity (3) is independent of (1)-(2), (4).

Proof. Let $B$ be the set of bi-identities given by (1)-(2), (4). All these biidentities $u=v$ have the following property: $\wedge$ is contained either in both of the terms $u, v$ or in none of them. Application of the rules (A)-(D) again yields such bi-identities. Furthermore, an existing wedge $\wedge$ cannot be removed in a term by applying rule (E). Let us assume that the bi-identity $u=v \in \bar{B}$ does not contain a wedge (on any side) but the bi-identity

$$
u\left(x \rightarrow p, x^{\prime} \rightarrow q\right)=v\left(x \rightarrow p, x^{\prime} \rightarrow q\right)
$$

does contain a wedge on the right-hand side. Suppose this wedge comes from $q$ which has been substituted for $x^{\prime}$. Suppose that $u\left(x \rightarrow p, x^{\prime} \rightarrow q\right)$ does not contain a wedge. Then $u$ does not contain the letter $x^{\prime}$. Now each bi-identity which holds in $\mathscr{L} \mathscr{S}$ has the following property: if one side contains a letter $x$ 
(respectively $x^{\prime}$ ), then the other side contains either $x$ or $x^{\prime}$ (or both letters). The reason for this is that a bi-identity which does not have this property does not hold in the $e$-variety of all semilattices. Since $\bar{B} \subseteq \rho(\mathscr{L} \mathscr{F}), u=v$ has this property, too. Hence $x$ occurs in $u$. Then $p$ cannot contain a wedge since otherwise $u\left(x \rightarrow p, x^{\prime} \rightarrow q\right)$ contains a wedge. But then, in the bi-identity $p=p q p$ the wedge occurs on the right-hand side whereas it does not occur on the left-hand side. In other words, if a bi-identity having a wedge on one side only is in $B_{n}$, then there must be such a bi-identity in $B_{n-1}$. Since in our case $B_{0}$ does not contain such bi-identities, the same is true for $\bar{B}$.

Lemma 3.6. The bi-identity (4) is independent of (1)-(3).

Proof. Let $S=<x, x^{\prime}, y, y^{\prime} \mid y=1=y^{\prime}, x^{2}=x,\left(x^{\prime}\right)^{2}=x^{\prime}, x=x x^{\prime} x$, $x^{\prime} x x^{\prime}=x^{\prime}>$ be the $2 \times 2$ rectangular band with an identity adjoined. Define a binary operation $*$ on $S$ by $a * b=a b$. Then the binary semigroup $(S, \cdot, *)$ satisfies the bi-identities (1)-(3) but does not satisfy (4).

Finally we summarize the preceding lemmas.

Theorem 3.7. The system of bi-identities (1)-(4) is independent.

\section{SUb- $e$-VARIETIES}

Given the bi-identities (1)-(4), certain sub- $e$-varieties of $\mathscr{L} \mathscr{F}$ can be described in a convenient fashion. Some of the following $e$-varieties will be characterized by the behaviour of the idempotents. Let $S$ be a locally inverse semigroup and $E=E(S)$ be the set of idempotents of $S$. Restriction of the sandwich operation $\wedge$ to $E$ yields a binary algebra $(E, \wedge)$, a so-called pseudosemilattice. Such algebras have been studied, for instance, by Meakin, Nambooripad, Pastijn, and Schein (see [11-21, 23]). In the following, the phrase "The $e$-variety $\mathscr{V}$ is defined by the bi-identitiy $u=v$ " actually means that $\mathscr{V}$ is defined by $u=v$, together with the bi-identities (1)-(4) of Theorem 3.2. Furthermore, terms sometimes will not be expressed in their canonical forms according to the description of $F$ by Theorem 2.2. In fact, we shall omit parantheses provided there is no danger of confusion.

Recall that an $E$-solid (regular) semigroup is a regular semigroup whose idempotent-generated subsemigroup $\langle E(S)\rangle$ is completely regular.

Theorem 4.1. The e-variety $\mathscr{E} \mathscr{S} \mathscr{L} \mathscr{S}$ of all $E$-solid locally inverse semigroups is defined by $\left(x x^{\prime} \wedge y y^{\prime}\right) \wedge z z^{\prime}=x x^{\prime} \wedge\left(y y^{\prime} \wedge z z^{\prime}\right)$.

Proof. The bi-identity in the statement of the theorem forces the sandwich operation to be associative when restricted to the pseudosemilattice $E(S)$. Let $S$ be an $E$-solid locally inverse semigroup. The idempotent-generated subsemigroup $\langle E(S)\rangle$ of $S$ is completely regular and thus is a normal band of groups (since each local submonoid of $\langle E(S)\rangle$ is a semilattice of groups). Clearly, $E(S)=E(\langle E(S)\rangle)$. The pseudosemilattice of idempotents of a normal band of groups $T$ can easily be seen to be isomorphic to the normal band $T / \mathscr{H}$. This follows from the fact that in a normal band of groups the sandwich element $x \wedge y$ coincides with the unique idempotent which is $\mathscr{H}$-related to $x y$. In particular $(E(S), \wedge)$ satisfies the associative law. Conversely, let $S$ be a locally inverse semigroup which is not $E$-solid. Let $\mathscr{V}$ be the $e$-variety generated by 
$S$. By Hall [7], $\mathscr{V}$ contains the $e$-variety of all combinatorial strict regular semigroups $\mathscr{C} \mathscr{S} \mathscr{R}$ and in particular the 5-element nonorthodox 0-simple semigroup $C_{2}$. The pseudosemilattice $E\left(C_{2}\right)$ does not satisfy the associative law (which can be seen directly or from [2, Corollary 4.4]).

$E$-solid locally inverse semigroups are thus characterized as the locally inverse semigroups having associative pseudosemilattices of idempotents. The condition that the sandwich operation is associative on the entire semigroup is much more restrictive.

Theorem 4.2. The e-variety $\mathscr{N B} \mathscr{G}$ of all normal bands of groups is defined by $x \wedge(y \wedge z)=(x \wedge y) \wedge z$.

Proof. As mentioned in the proof of the preceding theorem, in any normal band of groups, $x \wedge y=(x y)^{0}$, the $\mathscr{H}$-related idempotent of $x y$. From this, associativity of $\wedge$ in $\mathscr{N B} \mathscr{G}$ follows immediately. The least locally inverse $e$-variety which is not contained in $\mathscr{N O B} \mathscr{G}$ is the $e$-variety generated by $B_{2}$, the fiveelement combinatorial Brandt semigroup. We have to show that associativity of $\wedge$ fails in $B_{2}=\left\langle a, a^{\prime} \mid a a=0=a^{\prime} a^{\prime}, a=a a^{\prime} a, a^{\prime}=a^{\prime} a a^{\prime}\right\rangle$. Notice that in any inverse semigroup the sandwich operation can be expressed as $x \wedge y=$ $x x^{-1} y^{-1} y$. Substituting $x \rightarrow a, y \rightarrow a, z \rightarrow a^{\prime}$ we get $a \wedge\left(a \wedge a^{\prime}\right)=a a^{\prime} \neq 0$ whereas $(a \wedge a) \wedge a^{\prime}=0$.

In this way, many other $e$-varieties of locally inverse semigroups admit an equational description. For instance, the $e$-variety of all completely simple semigroups $\mathscr{C} \mathscr{S}$ is the greatest $e$-variety which does not contain the $e$-variety of all semilattices $\mathscr{S}$. Therefore, each (bi)identity which holds in $\mathscr{C} \mathscr{S}$ but not in $\mathscr{S}$ forms a basis for $\mathscr{C} \mathscr{S}$. Each (bi)identity which holds in $\mathscr{C} \mathscr{S}$ and does not have the same set of variables on both sides therefore can serve as a basis for $\mathscr{C} \mathscr{S}$. One such identity is $x \wedge y=(x \wedge z) \wedge y$. Next consider the class $\mathscr{S} \mathscr{R}$ of all strict regular semigroups, that is, all subdirect products of completely ( 0 )simple semigroups. It is well known (see Hall [6]) that a semigroup $S$ belongs to this class if and only if each local submonoid $e S e$ is Clifford, that is, has central idempotents. A typical element in the typical local submonoid $z S z^{\prime}$ can be written as $z y z^{\prime}$ whereas a typical idempotent in $z S z^{\prime}$ can be expressed as $z x z^{\prime} \wedge z x z^{\prime}$. Whence a basis for $\mathscr{S} \mathscr{R}$ is

$$
\left(z x z^{\prime} \wedge z x z^{\prime}\right) z y z^{\prime}=z y z^{\prime}\left(z x z^{\prime} \wedge z x z^{\prime}\right) \text {. }
$$

As another example we mention the class $\mathscr{G} \mathscr{F}$ of all generalized inverse semigroups (see Hall [7]). This is just the class of all locally inverse semigroups $S$ whose idempotents form a subsemigroup, whence one possibility of a defining bi-identity for $\mathscr{G} \mathscr{F}$ is $\left(x x^{\prime} y y^{\prime}\right)^{2}=x x^{\prime} y y^{\prime}$. Finally consider the $e$-variety $\mathscr{I}$ of all inverse semigroups. Since inverse semigroups are precisely regular semigroups with commuting idempotents, a basis of the bi-identities of $\mathscr{I}$ is $x x^{\prime} y y^{\prime}=y y^{\prime} x x^{\prime}$. On the other hand, inverse semigroups are also precisely the locally inverse semigroups whose pseudosemilattice of idempotents is actually a semilattice. From Schein [23] we know that a pseudosemilattice is a semilattice if and only if it is commutative. Whence the bi-identity $x x^{\prime} \wedge y y^{\prime}=y y^{\prime} \wedge x x^{\prime}$ is another possibility of a basis for $\rho(\mathscr{I})$. The details are easy and therefore are left to the reader. 


\section{REFERENCES}

1. K. Auinger, The bifree locally inverse semigroup on a set, J. Algebra 166 (1994), 630-650.

2. _ The word problem for the bifree combinatorial strict regular semigroup, Math. Proc. Cambridge Philos. Soc. 113 (1993), 519-533.

3. A. H. Clifford, The free completely regular semigroup on a set, J. Algebra 59 (1979), 434-451.

4. G. Grätzer, Universal algebra, Van Nostrand, New York, 1968.

5. T. E. Hall, Congruences and Green's relations on regular semigroups, Glasgow Math. J. 13 (1972), 167-175.

6. _ Identities for existence varieties of regular semigroups, Bull. Austral. Math. Soc. 40 (1989), 59-77.

7. _ Regular semigroups: amalgamation and the lattice of existence varieties, Algebra Universalis 29 (1991), 79-108.

8. _ A concept of variety for regular semigroups, Semigroup Theory, Proceedings of the Monash University Conference on Semigroup Theory in Honor of G. P. Preston (T. E. Hall, P. R. Jones, and J. C. Meakin, eds.), World Scientific, Singapore, 1991, pp. 101-116.

9. J. M. Howie, An introduction to semigroup theory, Academic Press, London, 1976.

10. J. Kadourek and M. B. Szendrei, A new approach in the theory of orthodox semigroups, Semigroup Forum 40 (1990), 257-296.

11. J. C. Meakin, Local semilattices on two generators, Semigroup Forum 24 (1982), 95-116.

12. __ The free local semilattice on a set, J. Pure Appl. Algebra 27 (1983), 263-275.

13. J. C. Meakin and F. Pastijn, The structure of pseudosemilattices, Algebra Universalis 13 (1981), 355-372.

14. $\ldots$, The free pseudosemilattice on two generators, Algebra Universalis 14 (1982), 297-309.

15. K. S. S. Nambooripad, Structure of regular semigroups, Mem. Amer. Math. Soc., vol. 224, Amer. Math. Soc., Providence, RI, 1979.

16. __ The natural partial order on a regular semigroup, Proc. Edinburgh Math. Soc. (2) 23 (1980), 249-260.

17. __ Pseudosemilattices and biordered sets I, Simon Stevin 55 (1981), 103-110.

18. __ Pseudosemilattices and biordered sets II, Simon Stevin 56 (1982), 143-160.

19. __ Pseudosemilattices and biordered sets III, Simon Stevin 56 (1982), 239-256.

20. F. Pastijn, Rectangular bands of inverse semigroups, Simon Stevin 56 (1982), 1-97.

21. _ The structure of pseudo-inverse semigroups, Trans. Amer. Math. Soc. 273 (1982), 631-655.

22. M. Petrich, Inverse semigroups, Wiley, New York, 1984.

23. B. M. Schein, Pseudosemilattices and pseudolattices, Izv. Vyssh. Uchebn. Zaved. Mat. 117 (1972), 81-94; English transl.Amer. Math. Soc. Transl. 119 (1983), 1-16.

24. M. B. Szendrei, Free *-orthodox semigroups, Simon Stevin 59 (1985), 175-201.

25. P. G. Trotter, Normal partitions of idempotents of regular semigroups, J. Austral. Math. Soc. 26 (1978), 110-114.

26. Y. T. Yeh, The existence of e-free objects in e-varieties of regular semigroups, Internat. J. Algebra Comput. 2 (1992), 471-484.

INSTitut Für Mathematik, Strudlhofgasse 4, A-1090 WiEn, Austria

E-mail address: A8131DATCAWIUNI11.EDVZ. UNIVIE. AC.AT 